\title{
ON PARSEVAL WAVELET FRAMES VIA MULTIRESOLUTION ANALYSES IN $H_{G}^{2}$
}

\author{
A. SAN ANTOLÍN
}

\begin{abstract}
We give a characterization of all Parseval wavelet frames arising from a given frame multiresolution analysis. As a consequence, we obtain a description of all Parseval wavelet frames associated with a frame multiresolution analysis. These results are based on a version of Oblique Extension Principle with the assumption that the origin is a point of approximate continuity of the Fourier transform of the involved refinable functions. Our results are written for reducing subspaces.
\end{abstract}

\section{Introduction, NOTATION AND BASIC DEFINITIONS}

We are interested in the study of methods for constructing tight wavelet frames in reducing subspaces of $L^{2}$. In this paper, we emphasis on tight wavelet frames can be constructed via multiresolution analyses and extension principles. Mallat [36] and Meyer [37] introduced the definition of multiresolution analysis (MRA) as a general method for constructing wavelets. In order to construct wavelet frames, the requirements on the definition of MRA were weakened. In this sense, the notion of a frame multiresolution analysis (FMRA) was formulated by Benedetto and $\mathrm{Li}$ [5] as a natural extension of MRA. Furthermore, a generalized multiresolution analysis (GMRA) was first introduced by Baggett, Medina and Merril [3] and Papadakis [39] independently, see also the paper by de Boor, DeVore and A. Ron [10]. Bownik and Rzeszotnik [9] show construction procedures for tight framelets and wavelets from an GMRA. The notions of MRA and FMRA in a reducing subspace of $L^{2}(\mathbb{R})$ are introduced by Li and Lian [31]. On $\mathbb{R}^{n}$ see [27]. Relationships of FMRA and MRA in reducing subspaces $L^{2}\left(\mathbb{R}^{n}\right)$ are studied by Li and Zhang [33]. Li and Zhou [35], [34] develop GMRA-based construction procedures of frame wavelet systems in reducing subspaces of $L^{2}\left(\mathbb{R}^{n}\right)$. Jia and $\mathrm{Li}[25]$ present a refinable function-based construction of affine frames systems.

Gripenberg [19] and Hernández and Weiss [24] proved a characterization of all orthonormal wavelets associated to a multiresolution analysis in terms of its wavelet dimension function. Any orthonormal wavelet is associated with a generalized multiresolution analysis was proved by Papadakis [40]. In the paper by Kim, Kim and Lim [29] (see also Kim, Kim, Lee and Lim [28]), characterizations of the Riesz wavelets which are associated with a multiresolution analysis were proved. A generalization of theses results are given by Bownik and Garrigós [8]. Note that characterizations of biorthogonal wavelets from biorthogonal multiresolution analysis are proved in [29], [8] (see also Calogero and Garrigós [12]). Zalik [48] introduced

Key words and phrases. Dilation matrix, Extension Principles, Fourier transform, refinable function, tight wavelet frame.

2010 Mathematics Subject Classification: 42C40, 42C15. 
the notion of Riesz wavelet obtained by a multiresolution analysis. Moreover, he gave necessary and sufficient conditions on a given Riesz wavelet to be obtained by a multiresolution analysis. Bownik [7] studied both the notion of Riesz basis associated with a generalized multiresolution analysis and Riesz basis obtained by a generalized multiresolution analysis and proved that these two notions are equivalent. Charazterizations of Parseval wavelet frames associated with generalized multiresolution analysis are proved by Baggett, Medina and Merrill [3] and by Bakić [4].

A slight different point of view for constructing wavelet frames was first proposed in the Unitary Extension Principle (UEP) by Ron and Shen [43] (see also [42]). The UEP leads to explicit constructions of tight wavelet frames generated by a refinable function. A more flexible way for constructing wavelet frames is the so called Oblique Extension Principle (OEP). The OEP was introduced by Daubechies, Han, Ron and Shen [17]. These extension principles have been developed by Benedetto and Treiber [6], Petukhov [41], Chui, He and Stöckler [14], Chui, He, Stöckler and Sun [15], Bownik and Rzeszotnik [9], Han [22], [21], Li and Zhou [35], Stavropoulos [47] Atreas, Melas and Stavropoulos [2], [1] and Li and Zhang [32]. Observe that in these papers, they proved that the obtained sufficient conditions are also necessary.

Extensive studies on multiresolution analysis and extension principles are enclosed, for instance, in [13] and [30].

We give a solution to the problem of characterizing all Parseval wavelet frames arising from a fix frame multiresolution analysis in reducing subspaces of $L^{2}\left(\mathbb{R}^{n}\right)$. As a consequence, we obtain a new description of all Parseval wavelet frames associated with a frame multiresolution analysis. The proofs of these results are based on the characterization of the scaling functions in [27] and on a version of Oblique Extension Principle with no regularity at the origin on the modulus of the Fourier transform of the involved refinable functions.

Versions of the OEP without any extra condition on refinable functions have already proved in the literature. The first one version of the OEP was proved by Han [22], [21], were the contexts is in distribution spaces. The assumption at the origin of the Fourier transform of involved refinable functions is a limit in sense of distributions. In the paper by $\mathrm{Li}$ and Zhang [32, Theorem 1.2], an OEP for frames in reducing subspaces of $L^{2}\left(\mathbb{R}^{d}\right)$ is proved. The authors use the condition $\lim _{j \rightarrow-\infty} \hat{\psi}_{0}\left(A^{* j}.\right)=1$ for almost every point of $\Omega$, an $A^{*}$-invariant set of $\mathbb{R}^{d}$. Perhaps, the main advance of our paper is the use of the classical notion of approximate continuity. This is why we emphasis on this notion. In particular, this concept helps us to get a bit achievement of the behaviour on a neighborhood of the origin of the Fourier transform of refinable functions associated to Parseval wavelet frames. To illustrate our results we show some examples of Parseval wavelet frames where the origin of its Fourier transform of is not a point of continuity. At the end of this manuscript we will see that the conditions used by Han, by Li and Zhang and the condition used here are of different nature.

Before formulating our results let us introduce some notation and definitions.

The sets of strictly positive integers, integers, real and complex numbers will be denoted by $\mathbb{N}, \mathbb{Z}, \mathbb{R}$ and $\mathbb{C}$ respectively. $\mathbb{T}^{n}=\mathbb{R}^{n} / \mathbb{Z}^{n}, n \geq 1$, and with some abuse of the notation we consider also that $\mathbb{T}^{n}$ is the unit cube $[0,1)^{n}$.

We will denote $B_{r}(\mathbf{y})=\left\{\mathbf{x} \in \mathbb{R}^{n}:|\mathbf{x}-\mathbf{y}|<r\right\}$, and will write $B_{r}$ if $\mathbf{y}$ is the origin. If $A: \mathbb{R}^{n} \rightarrow \mathbb{R}^{n}$ is a linear map, $A^{*}$ will mean the adjoint of $A$. With 
some abuse in the notation, if we write $A$ we also mean the corresponding matrix respect to the canonical basis. Moreover $d_{A}=|\operatorname{det} A|$. For a Lebesgue measurable set $E \subset \mathbb{R}^{n}, E^{c}=\mathbb{R}^{n} \backslash E$ and the Lebesgue measure of $E$ in $\mathbb{R}^{n}$ will be denoted by $|E|_{n}$. If $\mathbf{x} \in \mathbb{R}^{n}$ then $\mathbf{x}+E=\{\mathbf{x}+\mathbf{y}:$ for $\mathbf{y} \in E\}$. We will denote $A(E)=\left\{\mathbf{x} \in \mathbb{R}^{n}: \mathbf{x}=A(\mathbf{t})\right.$ for $\left.\mathbf{t} \in E\right\}$ and the volume of $E$ changes under $A$ according to $|A E|_{n}=d_{A}|E|_{n}$. The characteristic function of a set $E \subset \mathbb{R}^{n}$ will be denoted by $\chi_{E}$, i.e. $\chi_{E}(\mathbf{x})=1$ if $\mathbf{x} \in E$ and $\chi_{E}(\mathbf{x})=0$ otherwise.

If we write $L^{p}\left(\mathbb{R}^{n}\right), n \geq 1,1 \leq p \leq \infty$, we mean the usual Lebesgue space. If we write $f \in L_{l o c}^{p}\left(\mathbb{R}^{n}\right)$ we mean the linear space of all measurable functions such that $f \chi_{K} \in L^{p}\left(\mathbb{R}^{n}\right)$ for every $K \subset \mathbb{R}^{n}$ compact sets.

If we take $f \in L^{p}\left(\mathbb{T}^{n}\right)$ we will understand that $f$ is defined on the whole space $\mathbb{R}^{n}$ as a $\mathbb{Z}^{n}$-periodic function.

A key tool in the study of wavelet frames is the Fourier transform. Here, our convention is that if $f \in L^{1}\left(\mathbb{R}^{n}\right)$,

$$
\widehat{f}(\mathbf{x}):=\int_{\mathbb{R}^{n}} f(\mathbf{t}) e^{-2 \pi i \mathbf{t} \cdot \mathbf{x}} d \mathbf{t},
$$

where $\mathbf{t} \cdot \mathbf{x}$ denotes the usual inner product of vectors $\mathbf{t}$ and $\mathbf{x}$ in $\mathbb{R}^{n}$. The definition of Fourier transform is extended as usual form to functions in $L^{2}\left(\mathbb{R}^{n}\right)$.

A linear map $A$ is called expansive if all (complex) eigenvalues of $A$ have absolute value greater than 1 . If $A$ is invertible, we consider the operator $D_{A}$ on $L^{2}\left(\mathbb{R}_{n}\right)$ defined by $D_{A} f(\mathbf{t})=d_{A}^{1 / 2} f(A \mathbf{t})$. The translation of a function $f \in L^{2}\left(\mathbb{R}^{n}\right)$ by $\mathbf{b} \in \mathbb{R}^{n}$ will be denoted by $\tau_{\mathbf{b}} f(\mathbf{t})=f(\mathbf{t}-\mathbf{b})$. For a subspace $S$ of $L^{2}\left(\mathbb{R}^{d}\right)$,

$$
D_{A} S=\left\{D_{A}^{j} f: f \in S\right\}, \quad \text { and } \quad \tau_{\mathbf{b}} S=\left\{\tau_{\mathbf{b}} f: f \in S\right\} .
$$

If we write $D_{A}^{-1}$ we mean the operator $D_{A^{-1}}, D_{A}^{0}$ is the identity map and $D_{A}^{\ell}$, $\ell \in \mathbb{N}$, is the $\ell$-th composition of the operator $D_{A}$ with itself.

A measurable set $E \subset \mathbb{R}^{n}$ such that $A E=E$ is called an $A$ invariant set or an $A$-set. A shift-invariant subspace $S \subset L^{2}\left(\mathbb{R}^{n}\right)$ is called $A$-reducing if $D_{A} S=S$. If $G \subset \mathbb{R}^{n}$ is an $A^{*}$-set, we denote by $H_{G}^{2}$ the closed linear subspace of $L^{2}\left(\mathbb{R}^{n}\right)$ defined by

$$
H_{G}^{2}=\left\{f \in L^{2}\left(\mathbb{R}^{n}\right): \widehat{f}=\widehat{f} \chi_{G}\right\} .
$$

That $S$ is an $A$-reducing subspaces of $L^{2}\left(\mathbb{R}^{n}\right)$ if and only if $S$ is an $H_{G}^{2}$ for $G \subset \mathbb{R}^{n}$, an $A^{*}$-set, is proved by Dai, Diao, Gu and Han [16].

For a given $\phi \in L^{2}\left(\mathbb{R}^{n}\right)$, set

$$
[\phi, \phi](\mathbf{t})=\sum_{\mathbf{k} \in \mathbb{Z}^{n}}|\phi(\mathbf{t}+\mathbf{k})|^{2}
$$

and denote

$$
\mathcal{N}_{\phi}=\left\{\mathbf{t} \in \mathbb{R}^{n}:[\phi, \phi](\mathbf{t})=0\right\} .
$$

For a measurable function $f: \mathbb{R}^{n} \rightarrow \mathbb{C}$ the support of $f$ is defined to be $\operatorname{supp}(f)=$ $\left\{\mathbf{t} \in \mathbb{R}^{n} ; f(\mathbf{t}) \neq 0\right\}$.

The sets are defined modulo a null measurable set and we will understand some equations as almost everywhere on $\mathbb{R}^{n}$ or $\mathbb{T}^{n}$. Moreover, in order to shorten the notation, we will consider $0 / 0=0$ or $0(1 / 0)=0$ in some expressions where such an indeterminacy appears.

If $A: \mathbb{R}^{n} \rightarrow \mathbb{R}^{n}$ is an expansive linear invertible map such that $A\left(\mathbb{Z}^{n}\right) \subset \mathbb{Z}^{n}$, then the quotient groups $\mathbb{Z}^{n} / A\left(\mathbb{Z}^{n}\right)$ and $A^{-1}\left(\mathbb{Z}^{n}\right) / \mathbb{Z}^{n}$ are well defined. We will denote by $\Omega_{A} \subset \mathbb{Z}^{n}$ and $\Gamma_{A} \subset A^{-1}\left(\mathbb{Z}^{n}\right)$ a full collection of representatives of the cosets of 
$\mathbb{Z}^{n} / A\left(\mathbb{Z}^{n}\right)$ and $A^{-1}\left(\mathbb{Z}^{n}\right) / \mathbb{Z}^{n}$ respectively. Recall that there are exactly $d_{A}$ cosets of $\mathbb{Z}^{n} / A\left(\mathbb{Z}^{n}\right)$ (see $[20]$ ). Thus there are exactly $d_{A}$ cosets of $A^{-1}\left(\mathbb{Z}^{n}\right) / \mathbb{Z}^{n}$.

The theory of frames was introduced by Duffin and Schaeffer [18]. A sequence $\left\{\phi_{n}\right\}_{n=1}^{\infty}$ of elements in a separable Hilbert space $\mathbb{H}$ is a frame for $\mathbb{H}$ if there exist constants $C_{1}, C_{2}>0$ such that

$$
C_{1}\|h\|^{2} \leq \sum_{n=1}^{\infty}\left|\left\langle h, \phi_{n}\right\rangle\right|^{2} \leq C_{2}\|h\|^{2}, \quad \forall h \in \mathbb{H},
$$

where $\langle\cdot, \cdot\rangle$ denotes the inner product on $\mathbb{H}$. The constants $C_{1}$ and $C_{2}$ are called frame bounds. The definition implies that a frame is a complete sequence of elements of $\mathbb{H}$. A frame $\left\{\phi_{n}\right\}_{n=1}^{\infty}$ is tight if we may choose $C_{1}=C_{2}$, and if in fact $C_{1}=C_{2}=$ 1 , we will call the frame a Parseval frame. A sequence $\left\{h_{n}\right\}_{n=1}^{\infty}$ of elements in a Hilbert space $\mathbb{H}$ is a frame sequence if it is a frame for $\operatorname{span}\left\{h_{n}\right\}_{n=1}^{\infty}$.

Let $A: \mathbb{R}^{n} \rightarrow \mathbb{R}^{n}$ be an expansive linear map such that $A\left(\mathbb{Z}^{n}\right) \subset \mathbb{Z}^{n}$ and $G \subset \mathbb{R}^{n}$ be an $A^{*}$-set. Let $\Psi=\left\{\psi_{1}, \ldots, \psi_{N}\right\} \subset L^{2}\left(\mathbb{R}^{n}\right)$ be a set of functions, we call

$$
X_{\Psi}:=\left\{D_{A}^{j} \tau_{\mathbf{k}} \psi_{\ell}: j \in \mathbb{Z}, \mathbf{k} \in \mathbb{Z}^{d}, 1 \leq \ell \leq N\right\}
$$

the affine system generated by $\Psi$. The set of functions $\Psi$ is called a wavelet frame associated to $A$ for $H_{G}^{2}$, if the affine system (1) is a frame for $H_{G}^{2}$. In this case, the affine system (1) is usually called affine frame. When the context is clear we do not write "associated to $A$ for $H_{G}^{2}$ ". If this affine system is a tight frame for $H_{G}^{2}$ then $\Psi$ is called a tight wavelet frame or tight framelet for $H_{G}^{2}$. In particular, a tight wavelet frame with frame bounds equal to 1 is usually called a Parseval wavelet frame or a Parseval framelet for $H_{G}^{2}$. The functions $\psi_{\ell}, \ell=1, \ldots, N$ are called the generators of the wavelet frame.

Given a linear invertible map $A$ and $G \subset \mathbb{R}^{n}$ as above, by a frame multiresolution analysis associated to the dilation $A$ in $H_{G}^{2}\left(H_{G}^{2}\right.$-FMRA) we mean a sequence of closed subspaces $V_{j}, j \in \mathbb{Z}$, of the Hilbert space $H_{G}^{2}$ that satisfies the following conditions:

(i) $V_{j} \subset V_{j+1}$ for every $j \in \mathbb{Z}$;

(ii) $f \in V_{j}$ if and only if $D_{A} f \in V_{j+1}$ for every $j \in \mathbb{Z}$;

(iii) $\overline{\bigcup_{j \in \mathbb{Z}} V_{j}}=H_{G}^{2}$;

(iv) There exists a function $\phi \in V_{0}$, that is called scaling function, such that $V_{0}=\overline{\operatorname{span}}\left\{\tau_{\mathbf{k}} \phi: \mathbf{k} \in \mathbb{Z}^{n}\right\}$.

When the system $\left\{\tau_{\mathbf{k}} \phi: \mathbf{k} \in \mathbb{Z}^{n}\right\}$ is an orthonormal basis for $V_{0}$, then the $H_{G^{-}}^{2}$ FMRA is called an orthonormal multiresolution analysis or simply a multiresolution analysis in $H_{G}^{2}\left(H_{G}^{2}\right.$-MRA).

One of the possible ways for constructing an $H_{G}^{2}$-FMRA is to start with a scaling function $\phi \in H_{G}^{2}$. A function $\phi \in H_{G}^{2}$ generates an $H_{G}^{2}-F M R A$ if $V_{0}=$ $\overline{\operatorname{span}}\left\{\tau_{\mathbf{k}} \phi: \mathbf{k} \in \mathbb{Z}^{n}\right\}$ and the subspaces

$$
V_{j}=\overline{\operatorname{span}}\left\{D_{A}^{j} \tau_{\mathbf{k}} \phi ; \mathbf{k} \in \mathbb{Z}^{n}\right\}, \quad j \in \mathbb{Z}
$$

of the Hilbert space $H_{G}^{2}$ satisfy the conditions (i) and (iii).

If $\phi \in H_{G}^{2}$ generates an $H_{G}^{2}$-FMRA, then $\phi \in V_{0} \subset V_{1}$. Thus,

$$
\phi=\sum_{\mathbf{k} \in \mathbb{Z}^{n}} a_{\mathbf{k}} D_{A} \tau_{\mathbf{k}} \phi, \quad a_{\mathbf{k}} \in \mathbb{C},
$$


where the convergence is in $L^{2}\left(\mathbb{R}^{n}\right)$. Taking the Fourier transform, we can write

$$
\widehat{\phi}\left(A^{*} \mathbf{t}\right)=H(\mathbf{t}) \widehat{\phi}(\mathbf{t}) \quad \text { a.e. on } \mathbb{R}^{n}
$$

where $H$ is a $\mathbb{Z}^{n}$-periodic measurable function. A function that satisfies a equality as (3) is called refinable.

Given a multiresolution analysis, the following describes a standard procedure for constructing wavelet frames. If $\left\{V_{j}\right\}_{j \in \mathbb{Z}} \subset H_{G}^{2}$ is an $H_{G}^{2}$-FMRA, we denote by $W_{j}$ the orthogonal complement of $V_{j}$ in $V_{j+1}$. Thus, by condition (i), we have $V_{j+1}=W_{j} \oplus V_{j}$. Moreover, condition (iii) implies that $V_{j+1}=\oplus_{k<j} W_{k}$ and $H_{G}^{2}=\oplus_{j \in \mathbb{Z}} W_{j}$. Observe that if $\left\{\tau_{\mathbf{k}} \psi_{\ell}: \mathbf{k} \in \mathbb{Z}^{d}, 1 \leq \ell \leq N\right\}$ is a Parseval frame for $W_{0}$, then the system $\left\{D_{A}^{j} \tau_{\mathbf{k}} \psi_{\ell}: j \in \mathbb{Z}, \mathbf{k} \in \mathbb{Z}^{d}, 1 \leq \ell \leq N\right\}$ is a Parseval frame for $H_{G}^{2}$.

Definition 1. Let $\left\{V_{j}\right\}_{j \in \mathbb{Z}} \subset H_{G}^{2}$ be an $H_{G}^{2}-F M R A$. A Parseval wavelet frame $\Psi=$ $\left\{\psi_{1}, \ldots, \Psi_{N}\right\}$ for $H_{G}^{2}$ is said to be associated with $\left\{V_{j}\right\}_{j \in \mathbb{Z}}$ if $\left\{\tau_{\mathbf{k}} \psi_{\ell}: \mathbf{k} \in \mathbb{Z}^{d}, 1 \leq\right.$ $\ell \leq N\}$ is a Parseval frame for $W_{0}=V_{1} \ominus V_{0}$. We say that a Parseval wavelet frame is an $H_{G}^{2}-F M R A$ wavelet frame if $\Psi$ is Parseval wavelet frame associated with some $H_{G}^{2}-F M R A$.

A slight more flexible type of Parseval wavelet frames is the following.

Definition 2. Let $\left\{V_{j}\right\}_{j \in \mathbb{Z}} \subset H_{G}^{2}$ be an $H_{G}^{2}-F M R A$. We say that $\Psi=\left\{\psi_{1}, \ldots, \Psi_{N}\right\} \subset$ $H_{G}^{2}$ is a Parseval wavelet frame arising from $\left\{V_{j}\right\}_{j \in \mathbb{Z}}$ if $\Psi \subset V_{1}$ and the associated affine system (1) is a Parseval wavelet frame for $H_{G}^{2}$. Sometimes it is said that $\Psi=\left\{\psi_{1}, \ldots, \Psi_{N}\right\} \subset H_{G}^{2}$ is an $H_{G}^{2}-F M R A$ based wavelet frame if $\Psi$ is Parseval wavelet frame arising from some $H_{G}^{2}-F M R A$.

The following definitions were introduced in [27].

Definition 3. Let $A: \mathbb{R}^{n} \rightarrow \mathbb{R}^{n}$ be an expansive linear map and let $G \subset \mathbb{R}^{n}$ be an $A^{*}$-set. It is said that $\mathbf{x} \in \mathbb{R}^{n}$ is a point of $(A, G)$-density for a measurable set $E \subset \mathbb{R}^{n},|E|_{n}>0$, if for any $r>0$

$$
\lim _{j \rightarrow \infty} \frac{\left|E \cap\left[\left(G \cap A^{-j} B_{r}\right)+\mathbf{x}\right]\right|_{n}}{\left|G \cap A^{-j} B_{r}\right|_{n}}=1 .
$$

Definition 4. Let $A: \mathbb{R}^{n} \rightarrow \mathbb{R}^{n}$ be an expansive linear map and let $G \subset \mathbb{R}^{n}$ be an $A^{*}$-set. Let $f: \mathbb{R}^{n} \longrightarrow \mathbb{C}$ be a measurable function. It is said that $\mathbf{x} \in \mathbb{R}^{n}$ is a point of $(A, G)$-approximate continuity of the function $f$ if there exists $E \subset \mathbb{R}^{n}$, $|E|_{n}>0$, such that $\mathbf{x}$ is a point of $(A, G)$-density for the set $E$ and

$$
\lim _{\substack{\mathbf{y} \rightarrow \mathbf{x} \\ \mathbf{y} \in E}} f(\mathbf{y})=f(\mathbf{x})
$$

Observe that if $G=\mathbb{R}^{n}$ and $A=a I$, where $a>1$ and $I$ is the identity map on $\mathbb{R}^{n}$, then the definition of a point of $(A, G)$-approximate continuity coincides with the well-known definition of approximate continuity (cf. [38], [11]).

This paper is structured as follows. In Section 2, we write the main results of this paper with their proofs. In Section 3, we show some examples of Parseval wavelet frames. Section 4 is devoted to compare conditions on the Fourier transform of involved refinable functions in different versions of Extension Principles. 


\section{MAIN RESUlt}

We write the main results of this paper. In what follows, we fix $A: \mathbb{R}^{n} \rightarrow \mathbb{R}^{n}$ an expansive linear map such that $A\left(\mathbb{Z}^{n}\right) \subset \mathbb{Z}^{n}$. Let us fix $\Gamma_{A^{*}}=\left\{\mathbf{p}_{k}\right\}_{k=0}^{d_{A}-1}$, where $\mathbf{p}_{0}=\mathbf{0}$, a full collection of representatives of the cosets of $\left(A^{*}\right)^{-1} \mathbb{Z}^{n} / \mathbb{Z}^{n}$. Moreover, we fix $G \subset \mathbb{R}^{n}$, an $A^{*}$-set, and the subspace $H_{G}^{2}$. In order to shorten the notation, if we write a wavelet frame we mean a wavelet frame for $H_{G}^{2}$ associated to the dilation A.

The following result characterizes all Parseval wavelet frames for some reducing subspace that can be constructed via Oblique Extension Principle.

Theorem 1. Let $\phi \in H_{G}^{2}$ such that

$$
\widehat{\phi}\left(A^{*} \mathbf{t}\right)=H_{0}(\mathbf{t}) \widehat{\phi}(\mathbf{t}), \quad \text { a.e. },
$$

where $H_{0} \in L^{\infty}\left(\mathbb{T}^{n}\right)$. Let $H_{1}, \ldots, H_{N} \in L^{\infty}\left(\mathbb{T}^{n}\right)$ and define $\psi_{1}, \ldots, \psi_{N} \in L^{2}\left(\mathbb{R}^{n}\right)$ by

$$
\widehat{\psi_{\ell}}\left(A^{*} \mathbf{t}\right)=H_{\ell}(\mathbf{t}) \widehat{\phi}(\mathbf{t}) \quad \text { a.e. }, \quad \ell=1, \ldots, N .
$$

Then the following are equivalent:

i) The set of functions $\left\{\psi_{\ell}: \ell=1, \ldots, N\right\}$ is a Parseval wavelet frame for $H_{G}^{2}$.

ii) There exists $S$, a non-negative $\mathbb{Z}^{n}$-periodic measurable function such that $\sqrt{S}|\widehat{\phi}| \in H_{G}^{2}$ and also

(a) the origin is a point of $\left(A^{*}, G\right)$-approximate continuity for $S|\widehat{\phi}|^{2}$, provided $S(\mathbf{0})|\widehat{\phi}(\mathbf{0})|^{2}=1$;

(b)

$$
S\left(A^{*} \mathbf{t}\right)\left|H_{0}(\mathbf{t})\right|^{2}+\sum_{\ell=1}^{N}\left|H_{\ell}(\mathbf{t})\right|^{2}=S(\mathbf{t}) \quad \text { a.e. } \quad \mathbf{t} \in \mathbb{R}^{n} \backslash \mathcal{N}_{\widehat{\phi}} ;
$$

(c) the equality

$$
S\left(A^{*} \mathbf{t}\right) H_{0}(\mathbf{t}) \overline{H_{0}\left(\mathbf{t}+\mathbf{p}_{k}\right)}+\sum_{\ell=1}^{N} H_{\ell}(\mathbf{t}) \overline{H_{\ell}\left(\mathbf{t}+\mathbf{p}_{k}\right)}=0
$$

holds for a.e. $\mathbf{t} \in \mathbb{R}^{n} \backslash \mathcal{N}_{\widehat{\phi}}$ and for any $\mathbf{p}_{k}, k=1, \ldots, d_{A}-1$, such that $\mathbf{t}+\mathbf{p}_{k} \in \mathbb{R}^{n} \backslash \mathcal{N}_{\widehat{\phi}}$.

To prove $i$ i) implies $i$ ) in Theorem 1 we need the following result.

Lemma 1. Let $\phi \in H_{G}^{2}$ such that $|\widehat{\phi}(\mathbf{t})| \leq 1$ a.e. and the origin is a point of $\left(A^{*}, G\right)$-approximate continuity of $|\widehat{\phi}|$, provided $|\widehat{\phi}(\mathbf{0})|=1$. Let $g \in L^{2}\left(\mathbb{R}^{n}\right)$ such that $\widehat{g}$ is continuous and compactly supported. Consider $f \in L^{2}\left(\mathbb{R}^{n}\right)$ defined by $\widehat{f}=\widehat{g} \chi_{G}$. Then, for any $\varepsilon>0$ there exists $J \in \mathbb{N}$ such that

$$
(1-\varepsilon)\|f\|_{2}^{2} \leq \sum_{\mathbf{k} \in \mathbb{Z}^{n}}\left|\left\langle f, D_{A}^{j} \tau_{\mathbf{k}} \phi\right\rangle\right|^{2} \leq\|f\|_{2}^{2}, \quad \forall j \geq J .
$$


Proof. According to the hypotheses, $\operatorname{supp}(\widehat{f}) \subset B_{R}$ for a fix $R>0$ and there exists $K>0$ such that $|\widehat{f}(\mathbf{t})| \leq K$ for every $\mathbf{t} \in \mathbb{R}^{n}$. By Parseval's formula,

$$
\begin{aligned}
\sum_{\mathbf{k} \in \mathbb{Z}^{n}}\left|\left\langle f, D_{A}^{j} \tau_{\mathbf{k}} \phi\right\rangle\right|^{2} & =\sum_{\mathbf{k} \in \mathbb{Z}^{n}} \mid\left\langle\widehat{f}, \widehat{\left.D_{A}^{j} \tau_{\mathbf{k}} \phi\right\rangle\left.\right|^{2}}=\sum_{\mathbf{k} \in \mathbb{Z}^{n}}\left|\left\langle D_{A^{*}}^{j} \widehat{f}, \widehat{\tau_{\mathbf{k}} \phi}\right\rangle\right|^{2}\right. \\
& =\sum_{\mathbf{k} \in \mathbb{Z}^{d}}\left|\int_{\left(A^{*}\right)^{-j} B_{R}} D_{A^{*}}^{j} \widehat{f}(\mathbf{t}) \overline{\widehat{\phi}(\mathbf{t})} e^{2 \pi \mathbf{k} \cdot \mathbf{t}} d \mathbf{t}\right|^{2} .
\end{aligned}
$$

Since $A^{*}$ is expansive, there exists $j_{0} \in \mathbb{N}$ such that if $j \geq j_{0}$, then $\left(A^{*}\right)^{-j} B_{R} \subset$ $[-1 / 2,1 / 2]^{d}$. For those $j$, the sum over $\mathbf{k} \in \mathbb{Z}^{n}$ in (8) may be interpreted as the sum of the square of the modulus of the $-\mathbf{k}$-th Fourier coefficients of the function $D_{A^{* j}} \widehat{f}(\mathbf{t}) \overline{\widehat{\phi}(\mathbf{t})}$. Thus

(9) $\sum_{\mathbf{k} \in \mathbb{Z}^{n}}\left|\left\langle f, D_{A}^{j} \tau_{\mathbf{k}} \phi\right\rangle\right|^{2}=\int_{\left(A^{*}\right)^{-j} B_{R}}\left|D_{A^{* j}} \widehat{f}(\mathbf{t})\right|^{2}|\widehat{\phi}(\mathbf{t})|^{2} d \mathbf{t} \quad \forall j \geq j_{0}$.

Since $|\widehat{\phi}(\mathbf{t})| \leq 1$ a.e., the right inequality of (7) follows. Now, we prove the left inequality of (7).

Let $0<\varepsilon<1$ and take the set $\Lambda_{\varepsilon}=\left\{\mathbf{t} \in G:|\widehat{\phi}(\mathbf{t})| \leq 1-\frac{\varepsilon}{2}\right\}$. Since $|\widehat{\phi}(\mathbf{0})|=1$, $|\widehat{\phi}(\mathbf{t})| \leq 1$ a.e. and the origin is a point of $\left(A^{*}, G\right)$-approximate continuity of $|\widehat{\phi}|$, then

$$
\lim _{j \rightarrow \infty} \frac{\left|\left(G \cap\left(A^{*}\right)^{-j} B_{R}\right) \cap \Lambda_{\varepsilon}^{c}\right|_{n}}{\left|G \cap\left(A^{*}\right)^{-j} B_{R}\right|_{n}}=1 .
$$

This implies that there exists $J \geq j_{0}$ such that if $j \geq J$, we have

Thus, if $j \geq J$

$$
\left|\left(A^{*}\right)^{-j}\left(G \cap B_{R} \cap A^{* j} \Lambda_{\varepsilon}\right)\right|_{n}=\left|\left(G \cap\left(A^{*}\right)^{-j} B_{R}\right) \cap \Lambda_{\varepsilon}\right|_{n}<\frac{\varepsilon}{2 K^{2} d_{A}^{j}}\|f\|_{2}^{2} .
$$

$$
\left|G \cap B_{R} \cap A^{* j} \Lambda_{\varepsilon}\right|_{n}<\frac{\varepsilon}{2 K^{2}}\|f\|_{2}^{2} .
$$

According to (9), if $j \geq J$ we obtain

$$
\begin{aligned}
\sum_{\mathbf{k} \in \mathbb{Z}^{n}}\left|\left\langle f, D_{A}^{j} \tau_{\mathbf{k}} \phi\right\rangle\right|^{2} & \geq\left(1-\frac{\varepsilon}{2}\right) \int_{G \cap\left(A^{*}\right)^{-j} B_{R} \cap \Lambda_{\varepsilon}^{c}}\left|D_{A^{*}} \widehat{f}(\mathbf{t})\right|^{2} d \mathbf{t} \\
& \geq\left(1-\frac{\varepsilon}{2}\right)\|f\|_{2}^{2}-\int_{G \cap B_{R} \cap A^{* j} \Lambda_{\varepsilon}}|\widehat{f}(\mathbf{t})|^{2} d \mathbf{t} .
\end{aligned}
$$

Furthermore, by the inequality (10), if $j \geq J$ we have

$$
\begin{aligned}
\sum_{\mathbf{k} \in \mathbb{Z}^{n}}\left|\left\langle f, D_{A}^{j} \tau_{\mathbf{k}} \phi\right\rangle\right|^{2} & \geq\left(1-\frac{\varepsilon}{2}\right)\|f\|_{2}^{2}-K^{2}\left|G \cap B_{R} \cap A^{* j} \Lambda_{\varepsilon}\right|_{n} \\
& \geq\left(1-\frac{\varepsilon}{2}\right)\|f\|_{2}^{2}-K^{2} \frac{\varepsilon}{2 K^{2}}\|f\|_{2}^{2}=(1-\varepsilon)\|f\|_{2}^{2} .
\end{aligned}
$$

This finishes the proof.

Proof of Theorem 1. We prove $i$ ) implies $i$ ). Without loss in generality, we can assume that $H_{\ell}(\mathbf{t})=0$, a.e. $\mathbf{t} \in \mathcal{N}_{\widehat{\phi}}, \ell=0,1, \ldots, N$. The following

$$
\Theta(\mathbf{t})=\sum_{m=0}^{\infty} \sum_{\ell=1}^{N}\left|H_{\ell}\left(A^{* m} \mathbf{t}\right)\right|^{2} \prod_{k=0}^{m-1}\left|H_{0}\left(A^{* k} \mathbf{t}\right)\right|^{2},
$$


with the convention $\prod_{k=0}^{-1}\left|Q_{0}\left(A^{* k} \mathbf{t}\right)\right|^{2}=1$, is usually called fundamental function associated to $H_{0}, \ldots, H_{N}$. Let us mention that we have assumed $H_{\ell}(\mathbf{t})=0$ a.e. $\mathbf{t} \in \mathcal{N}_{\widehat{\phi}}$ to avoid problems with the measurability of $\Theta$. Note that fundamental functions were introduced in [43].

We will see that the conditions in $i$ ) hold when we consider $\Theta$ instead of $S$. According to Lemma 2.3 and Lemma 2.10 in [32], we know that

$$
1=\lim _{J \rightarrow-\infty} \Theta\left(A^{* J} \mathbf{t}\right)\left|\widehat{\phi}\left(A^{* J} \mathbf{t}\right)\right|^{2} \quad \text { a.e. } \quad \mathbf{t} \in G .
$$

Thus, the origin is a point of $\left(A^{*}, G\right)$-approximate continuity of $\Theta|\widehat{\phi}|^{2}$, if we set $\Theta(\mathbf{0})|\widehat{\phi}(\mathbf{0})|^{2}=1$, follows by [46, Lemma 2.5].

The remainder is proved in [32] (e.g. see also [2])

We prove $i$ ) implies $i$ ). First, we prove this implication in the particular case when $S(\mathbf{t})=1$. This case is usually called Unitary Extension Principle.

Our starting point is Lemma 1. Afterwards, the proof may be finishes following the strategy by Benedetto and Treiber [6] (see also [32], [30]).

When $S$ is a general function described in $i i)$, consider $\varphi \in H_{G}^{2}$ defined by $\widehat{\varphi}(\mathbf{t})=\sqrt{S(\mathbf{t})} \widehat{\phi}(\mathbf{t})$. Observe that

$$
\widehat{\varphi}\left(A^{*} \mathbf{t}\right)=\sqrt{S\left(A^{*} \mathbf{t}\right)} \widehat{\phi}\left(A^{*} \mathbf{t}\right)=\sqrt{S\left(A^{*} \mathbf{t}\right)} H_{0}(\mathbf{t}) \widehat{\phi}(\mathbf{t})=Q_{0}(\mathbf{t}) \widehat{\varphi}(\mathbf{t}) \quad \text { a.e. }
$$
and

$$
\widehat{\psi_{\ell}}\left(A^{*} \mathbf{t}\right)=\sqrt{\frac{1}{S(\mathbf{t})}} H_{\ell}(\mathbf{t}) \sqrt{S(\mathbf{t})} \widehat{\phi}(\mathbf{t})=Q_{\ell}(\mathbf{t}) \widehat{\varphi}(\mathbf{t}), \quad \text { a.e. } \quad \ell=1, \cdots, N,
$$

where $Q_{0}, Q_{1} \ldots, Q_{N}$ are $\mathbb{Z}^{n}$-periodic measurable functions defined by

$$
Q_{0}(\mathbf{t})=\sqrt{\frac{S\left(A^{*} \mathbf{t}\right)}{S(\mathbf{t})}} H_{0}(\mathbf{t}), \quad Q_{\ell}(\mathbf{t})=\sqrt{\frac{1}{S(\mathbf{t})}} H_{\ell}(\mathbf{t}), \quad \ell=1, \ldots, N .
$$

According to our hypotheses, the origin is a point of $\left(A^{*}, G\right)$-approximate continuity for $\widehat{\varphi}$, provided that $\widehat{\varphi}(\mathbf{0})=1$. Analogously to the proof of Proposition 1.11 in [17], we get that $\varphi, \psi_{\ell}, Q_{0}$ and $Q_{\ell}, \ell=1, \ldots, N$, satisfy the Unitary Extension Principles. Then the statement follows.

The following result gives a characterization of all Parseval wavelet frames for an $H_{G}^{2}$ arising from a fix $H_{G}^{2}$-FMRA.

Theorem 2. Let $\left\{V_{j}\right\}_{j \in \mathbb{Z}} \subset H_{G}^{2}$ be an $H_{G}^{2}$-FMRA with a scaling function $\phi$. Let $\varphi \in H_{G}^{2}$ defined by $\widehat{\varphi}(\mathbf{t})=\widehat{\phi}(\mathbf{t}) /[\widehat{\phi}, \widehat{\phi}]^{1 / 2}(\mathbf{t})$. Let $\Psi=\left\{\psi_{1}, \ldots, \psi_{N}\right\} \subset H_{G}^{2}$. The following are equivalent.

i) The set of functions $\Psi$ is a Parseval wavelet frame for $H_{G}^{2}$ arising from $\left\{V_{j}\right\}_{j \in \mathbb{Z}}$.

ii) There exist $H_{0}, H_{1}, \ldots, H_{N} \in L^{\infty}\left(\mathbb{T}^{n}\right)$ such that a)

$$
\begin{gathered}
\widehat{\varphi}\left(A^{*} \mathbf{t}\right)=H_{0}(\mathbf{t}) \widehat{\varphi}(\mathbf{t}), \quad \text { a.e. }, \\
\widehat{\psi_{\ell}}\left(A^{*} \mathbf{t}\right)=H_{\ell}(\mathbf{t}) \widehat{\varphi}(\mathbf{t}) \quad \text { a.e., } \quad \ell=1, \ldots, N,
\end{gathered}
$$

and

b) there exists a non-negative $S \in L^{\infty}\left(\mathbb{T}^{n}\right)$ such that the origin is a point of $\left(A^{*}, G\right)$-approximate continuity for $S$ if we set $S(\mathbf{0})=1$, and the equalities in (5) and (6) are satisfied. 
Proof. We see that i) implies ii). There exist $H_{0}, H_{1}, \ldots, H_{N}$ some $\mathbb{Z}^{n}$-periodic measurable functions such that

$$
\widehat{\varphi}\left(A^{*} \mathbf{t}\right)=H_{0}(\mathbf{t}) \widehat{\varphi}(\mathbf{t}) \quad \text { a.e., and } \widehat{\psi_{\ell}}\left(A^{*} \mathbf{t}\right)=H_{\ell}(\mathbf{t}) \widehat{\varphi}(\mathbf{t}), \quad \ell=1, \ldots, N \quad \text { a.e. }
$$

because $\left\{\psi_{1}, \ldots, \psi_{N}\right\} \subset V_{1}$. Since $\left\{\varphi(\cdot-\mathbf{k}): \mathbf{k} \in \mathbb{Z}^{n}\right\}$ is a Parseval frame for $V_{0}$, we have $\sum_{\mathbf{k} \in \mathbb{Z}^{n}}|\widehat{\varphi}(\mathbf{t}-\mathbf{k})|^{2}=1$ a.e. $\mathbf{t} \in \mathbb{T}^{n} \backslash \mathcal{N}_{\widehat{\varphi}}$. Thus $H_{0}$ can be taken in $L^{\infty}\left(\mathbb{T}^{n}\right)$. Indeed, it can be assumed that $H_{\ell}(\mathbf{t})=0, \ell=0,1, \ldots, N$, a.e. on $\mathcal{N}_{\widehat{\varphi}}$.

Let $\Theta$ be the fundamental function associated to $H_{0}, H_{1}, \ldots, H_{N}$ defined as in (11). Since $\Psi$ is a Parseval frame for $H_{G}^{2}$, we have already said that $\Theta$ satisfies the condition ii) in Theorem 1 if we consider $\Theta$ instead of $S$. It remains to see that $\Theta, H_{1}, \ldots, H_{N}$ are in $L^{\infty}\left(\mathbb{T}^{n}\right)$, and the origin is a point of $\left(A^{*}, G\right)$-approximate continuity for $\Theta$ is we set $\widehat{\Theta}(\mathbf{0})=1$.

We know that $\Theta(\mathbf{t}) \sum_{\mathbf{k} \in \mathbb{Z}^{n}}|\widehat{\phi}(\mathbf{t}-\mathbf{k})|^{2} \in L^{\infty}\left(\mathbb{T}^{n}\right)$ (e.g. see (2.4) in [2] or proof of Lema 2.12 in [32]). Furthermore, since $\sum_{\mathbf{k} \in \mathbb{Z}^{n}}|\widehat{\varphi}(\mathbf{t}-\mathbf{k})|^{2}=1$ a.e. $\mathbf{t} \in \mathbb{T}^{n} \backslash \mathcal{N}_{\widehat{\varphi}}$ and bearing in mind that $\Theta(\mathbf{t})=0$ a.e. on $\mathcal{N}_{\hat{\varphi}}$, we conclude that $\Theta$ is in $L^{\infty}\left(\mathbb{T}^{n}\right)$. Hence $H_{1}, \ldots, H_{N}$ can be taken in $L^{\infty}\left(\mathbb{T}^{n}\right)$.

By ii) in Theorem 1 we know that the origin is a point of $\left(A^{*}, G\right)$-approximate continuity for $\Theta|\widehat{\varphi}|^{2}$ is we set $\Theta(\mathbf{0})|\widehat{\varphi}(\mathbf{0})|^{2}=1$. By Theorem 3.1 in [27], the origin is a point of $\left(A^{*}, G\right)$-approximate continuity for $\widehat{\varphi}$ is we set $\widehat{\varphi}(\mathbf{0})=1$. Hence, the origin is a point of $\left(A^{*}, G\right)$-approximate continuity for $\Theta$ is we set $\Theta(\mathbf{0})=1$ follows.

We check that $i$ ) implies $i$ ). By $a$ ) we know that $\Psi \subset V_{1}$. In addition, by Theorem 1, we conclude that $\Psi$ is a Parseval wavelet frame for $H_{G}^{2}$. Hence the proof is finished.

The following is a characterization of all Parseval wavelet frames associated with a fix $H_{G}^{2}$-FMRA.

Corollary 1. Let $\left\{V_{j}\right\}_{j \in \mathbb{Z}} \subset H_{G}^{2}$ be an $H_{G}^{2}$-FMRA with a scaling function $\phi$. Let $\varphi \in L^{2}\left(\mathbb{R}^{n}\right)$ defined by $\widehat{\varphi}(\mathbf{t})=\widehat{\phi}(\mathbf{t}) /[\widehat{\phi}, \widehat{\phi}]^{1 / 2}(\mathbf{t})$. Let $\Psi=\left\{\psi_{1}, \ldots, \psi_{N}\right\} \subset H_{G}^{2}$. The following are equivalent.

$\alpha)$ The set of functions $\Psi$ is a Parseval wavelet frame for $H_{G}^{2}$ associated with $\left\{V_{j}\right\}_{j \in \mathbb{Z}}$.

$\beta$ ) The condition ii) in Theorem 2 holds and also

(12) $\sum_{k=0}^{d_{A}-1} H_{0}\left(\mathbf{t}+\mathbf{p}_{k}\right) \overline{H_{\ell}\left(\mathbf{t}+\mathbf{p}_{k}\right)}=0, \quad$ for a.e. $\mathbf{t} \in \mathbb{R}^{n} \backslash \mathcal{N}_{\widehat{\phi}}, \quad \ell=\{1,2, \ldots, N\}$.

Proof. First, observe that the condition (12) means that $\psi_{i}$ are orthogonal to the subspace $V_{0}$ (e.g. see the proof of Theorem 2.4.3 in [30]). Therefore, by Theorem $2,(12)$ and the definition of $W_{j}, j \in \mathbb{Z}$, the statements follow.

\section{EXAmples}

The main results in this note focus on Parseval wavelet frames with no regularity of the Fourier transform at the origin. Here, we write some examples to illustrate Theorem 1 and 2 .

The following example shows orthonormal wavelets such that its Fourier transform is not continuous at the origin.

Example 1. Let $A: \mathbb{R}^{n} \rightarrow \mathbb{R}^{n}$ be an expansive linear map such that $A \mathbb{Z}^{n} \subset \mathbb{Z}^{n}$ and $G=\mathbb{R}^{n}$. Our starting point is $\phi \in L^{2}\left(\mathbb{R}^{n}\right)$, the scaling function of a multiresolution analysis associated to $A$ that is constructed in [44, Theorem 2.2]. This 
function $\phi$ is defined by $\widehat{\phi}=\chi_{E}$, where $E \subset \mathbb{R}^{n}$ is a bounded measurable set that satisfies

i) The origin is a point of $A^{*}$-density for $E$ and $\mathbf{0} \in E$;

ii) $\sum_{\mathbf{k} \in \mathbb{Z}^{n}} \chi_{E}(\mathbf{t})=1$;

iii) $\left(A^{*}\right)^{-1} E \subset E$;

$i v)$ for any open neighborhood of the origin, $U$, there exists an open set included in $E^{c} \cap U$.

By the condition ii), we have that $\left\{\phi(\cdot-\mathbf{k}): \mathbf{k} \in \mathbb{Z}^{n}\right\}$ is an orthonormal system. Acording to $i$ ) and $i$ ii), the function

$$
H_{0}(\mathbf{t})=\sum_{\mathbf{k} \in \mathbb{Z}^{n}} \frac{\chi_{E}\left(A^{*}(\mathbf{t}+\mathbf{k})\right)}{\chi_{E}(\mathbf{t}+\mathbf{k})}
$$

is a well defined $\mathbb{Z}^{n}$-periodic such that it satisfies the following refinable equation

$$
\widehat{\phi}\left(A^{*} \mathbf{t}\right)=H_{0}(\mathbf{t}) \widehat{\phi}(\mathbf{t}) \text {. }
$$

If $\left\{\mathbf{p}_{k}\right\}_{k=0}^{d_{A}-1}$, where $\mathbf{p}_{0}=\mathbf{0}$, a full collection of representatives of the cosets of $\left(A^{*}\right)^{-1} \mathbb{Z}^{n} / \mathbb{Z}^{n}$, define

$$
\widehat{\psi_{\ell}}\left(A^{*} \mathbf{t}\right)=H_{0}\left(\mathbf{t}+\mathbf{p}_{\ell}\right) \widehat{\phi}(\mathbf{t}), \quad \ell \in\left\{1, \ldots, d_{A}-1\right\} .
$$

It is not hard to prove that $\Psi=\left\{\psi_{1}, \ldots, \psi_{d_{A}-1}\right\} \subset L^{2}\left(\mathbb{R}^{n}\right)$ is a set of orthonormal wavelets in $L^{2}\left(\mathbb{R}^{n}\right)$ associated to $A$. In addition, according to the conditions iii) and $i v)$, some of $\widehat{\psi_{\ell}}$ are not continuous at the origin.

For any $H_{G}^{2}$ where $G$ is open, we construct Parseval wavelet frames where the Fourier transform of some generators is not continuous at the origin.

Example 2. Let $A: \mathbb{R}^{n} \rightarrow \mathbb{R}^{n}$ be an expansive linear map such that $A \mathbb{Z}^{n} \subset \mathbb{Z}^{n}$ and $G \subset$ be an open $A^{*}$-set.

Since $A^{*}$ is expansive, $\exists R>0$ such that $\bigcup_{j=0}^{\infty}\left(A^{*}\right)^{-j} B_{R} \subset\left[-\frac{1}{2}, \frac{1}{2}\right)^{n}$. Let $F:=G \cap \bigcup_{j=0}^{\infty}\left(A^{*}\right)^{-j} B_{R}$ and observe that the set $F$ can be written by $F=$ $G \cap \bigcup_{j=0}^{j_{0}}\left(A^{*}\right)^{-j} B_{R}$ where $j_{0} \in \mathbb{N}$ is such that if $j \geq j_{0}$ then $\left(A^{*}\right)^{-j} B_{R} \subset B_{R}$. This $j_{0}$ exists because $A^{*}$ is an expansive linear map. Further, the set $F \backslash\left(A^{*}\right)^{-1}(\bar{F})$ is an open measurable set because the set $F$ is an open measurable set and $A^{*}$ is a continuous map. Moreover, since $A^{*}$ is expansive, it is clear that $\left|F \backslash\left(A^{*}\right)^{-1}(\bar{F})\right|_{n}>0$.

Now, let $F_{l}=\left(A^{*}\right)^{-l} F \backslash\left(A^{*}\right)^{-l-1} F, l \in\{0,1,2, \ldots\}$. Observe that the measurable sets $F_{l}$ are disjoint. Moreover, $F \backslash\{\mathbf{0}\}=\bigcup_{l=0}^{\infty} F_{l}$. If $\mathbf{x} \in F \backslash\{\mathbf{0}\}$, then $\mathbf{x} \in$ $\left(G \cap\left(A^{*}\right)^{-j} B_{R}\right)$ for some $j \in\{0,1,2, \ldots\}$. Let $j_{1}$ the biggest number in $\{0,1,2, \ldots\}$ such that $\mathbf{x} \in\left(G \cap\left(A^{*}\right)^{-j_{1}} B_{R}\right)$. Thus $\mathbf{x} \in F_{j_{1}}=\left(A^{*}\right)^{-j_{1}} F \backslash\left(A^{*}\right)^{-j_{1}-1} F$.

Furthermore, observe that $\exists \mathbf{y}_{\mathbf{0}} \in \mathbb{R}^{n}$ and $\exists r>0$ such that $B_{r}+\mathbf{y}_{\mathbf{0}} \subset F_{0}$.

Let

$$
E=\bigcup_{j=0}^{\infty} F_{j} \backslash\left[\left(A^{*}\right)^{-j} B_{2^{-j} r}+\mathbf{y}_{\mathbf{0}}\right] .
$$

It is easy to see that the set $E$ satisfies $|E|_{n}>0, E \subset\left[-\frac{1}{2}, \frac{1}{2}\right)^{n},\left(A^{*}\right)^{-1} E \subset$ and for any open neighborhood of the origin, $U$, there exists an open set included in $E^{c} \cap U$. In addition, the origin is a point of $\left(A^{*}, G\right)$-density for $E$ can be proved as in Step 2 of the proof of Theorem 2.2 in [44]. 
From this set E, we continue our construction as in Example 1 to obtain $\Psi=$ $\left\{\psi_{1}, \ldots, \psi_{d_{A}-1}\right\} \subset H_{G}^{2}$, a set of Parseval wavelet frames for $H_{G}^{2}$, where the restriction on the set $G$ of some $\widehat{\psi_{\ell}}$ are not continuous at the origin.

The purpose of the following example is to show visually how the previous examples works.

Example 3. Let $M$ be the linear map in $\mathbb{R}^{2}$ such that $M^{*}(x, y)=(2 x+2 y, 2 y)$. It is easy to see that

$$
\left\{\mathbf{p}_{0}=(0,0), \quad \mathbf{p}_{1}=\left(\frac{1}{2}, 0\right), \quad \mathbf{p}_{2}=\left(0, \frac{1}{2}\right), \quad \mathbf{p}_{3}=\left(\frac{1}{2}, \frac{1}{2}\right)\right\}
$$

is a full collection of representatives of the cosets of $\left(M^{*}\right)^{-1} \mathbb{Z}^{2} / \mathbb{Z}^{2}$.

Take the set $E=\left[-\frac{1}{2}, \frac{1}{2}\right]^{2} \backslash\left\{(x, y) \in\left(-\frac{1}{2}, \frac{1}{2}\right)^{2}: x, y>0\right\}$. The function $\left.\phi \in L^{(} \mathbb{R}^{2}\right)$ defined by $\widehat{\phi}=\chi_{E}$ is a scaling function of a frame multiresolution analysis associated to $M$.
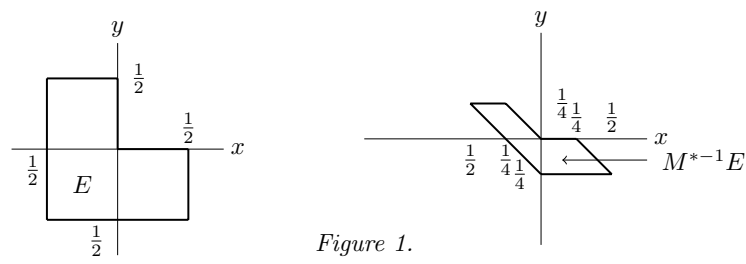

Define $H_{0}$ as the $\mathbb{Z}^{2}$-periodization of $\chi_{M^{*-1} E}$. Then $\left\{\psi_{1}, \psi_{2}, \psi_{3}\right\}$ defined by

$$
\widehat{\psi_{\ell}}\left(M^{*} \mathbf{t}\right)=H_{0}\left(\mathbf{t}+\mathbf{p}_{\ell}\right)(\mathbf{t}) \widehat{\phi}(\mathbf{t})
$$

is a Parseval wavelet frame for $L^{2}\left(\mathbb{R}^{2}\right)$ associated to the dilation $M$.

Observe that in Theorem 1, the condition that the origin must be a point of approximate continuity of the Fourier transform of the involve refinable function, when the function takes the value 1 at the origin, is not assumed. Then Theorem 1 provides a more flexible way for constructing wavelets frames than the different versions of the Oblique Extension Principle that appear in the literature. To show this fact, we write the following example.

Example 4. With the same notation in Example 1, let $C \in(0, \infty) \backslash\{1\}$. We take the function $\varphi:=C \phi$. It is clear that the origin is a point of $A^{*}$-approximate continuity of $\widehat{\varphi}$ with $\widehat{\varphi}(\mathbf{0})=C$ and

$$
\widehat{\varphi}\left(A^{*} \mathbf{t}\right)=C \widehat{\phi}\left(A^{*} \mathbf{t}\right)=C H_{0}(\mathbf{t}) \widehat{\phi}(\mathbf{t})=H_{0}(\mathbf{t}) \widehat{\varphi}(\mathbf{t}) .
$$

Moreover, for $\ell \in\left\{1, \ldots, d_{A}-1\right\}$ we can write

$$
\widehat{\psi_{\ell}}\left(A^{*} \mathbf{t}\right)=\frac{1}{C} H_{0}\left(\mathbf{t}+\mathbf{p}_{\ell}\right) \widehat{\varphi}(\mathbf{t})=Q_{\ell}(\mathbf{t}) \widehat{\varphi}(\mathbf{t}),
$$

where $Q_{\ell}(\mathbf{t})=\frac{1}{C} H_{0}\left(\mathbf{t}+\mathbf{p}_{\ell}\right)$. Therefore, the hypotheses and condition ii) in Theorem 1 hold for $\varphi, \psi_{\ell}$ the filters $H_{0}, Q_{\ell}$ and $S(\mathbf{t})=\frac{1}{c^{2}}$.

\section{ON CONDITION AT THE ORIGIN OF THE FOURIER TRANSFORM OF THE} REFINABLE FUNCTION.

In this last section, we will made some comments on the conditions at the origin of the Fourier Transform of an involved refinable function in Extension Principles 
assumed by Han [22], [21], by Li and Zhang [32] and the condition used in this note. In particular, we will see that those conditions are not equivalent.

The linear space of all compactly supported $C^{\infty}\left(\mathbb{R}^{n}\right)$ (test) functions with the usual topology will be denoted by $\mathcal{D}\left(\mathbb{R}^{n}\right)$. For $g \in \mathcal{D}\left(\mathbb{R}^{n}\right)$ and $f \in L_{l o c}^{1}\left(\mathbb{R}^{n}\right)$, with some abuse in the notation, we will use

$$
\langle f, g\rangle=\int_{\mathbb{R}^{n}} f(\mathbf{t}) \overline{g(\mathbf{t})} d \mathbf{t} .
$$

According to our context, the condition used by Han may be written as follows. Given $f \in L_{l o c}^{1}\left(\mathbb{R}^{n}\right)$, the following identity holds in the sense of distributions:

$$
\lim _{j \rightarrow \infty}\left|f\left(A^{-j} \mathbf{t}\right)\right|=1 \text {, }
$$

more precisely,

$$
\lim _{j \rightarrow \infty}\left\langle\left|f\left(A^{-j} \mathbf{t}\right)\right|, g\right\rangle=\langle 1, g\rangle, \quad \forall g \in \mathcal{D}\left(\mathbb{R}^{n}\right) .
$$

Let $f$ be the function in $L_{l o c}^{1}(\mathbb{R})$ defined by

$$
f(x)=\chi_{[-1,1]}(x)+\sum_{\ell=0}^{\infty} 2^{\ell+2} \chi_{\left(2^{-\ell-1}, 2^{-\ell-1}+2^{-2 \ell-2}\right)}(x),
$$

We will see that the origin is a point of approximate continuity of $f$ but (14) is not satisfied with $A=2$.

Denote $F=[-1,1] \backslash \bigcup_{\ell=0}^{\infty}\left(2^{-\ell-1}, 2^{-\ell-1}+2^{-2 \ell-2}\right)$. Since

$$
\begin{aligned}
\lim _{j \rightarrow \infty} \frac{\left|2^{-j}[-1,1] \cap F\right|}{\left|2^{-j}[-1,1]\right|} & =1-\lim _{j \rightarrow \infty} \frac{\left|2^{-j}[-1,1] \cap\left(\bigcup_{\ell=0}^{\infty}\left(2^{-\ell-1}, 2^{-\ell-1}+2^{-2 \ell-2}\right)\right)\right|}{\left|2^{-j}[-1,1]\right|} \\
& =1-\lim _{j \rightarrow \infty} \frac{\left|\bigcup_{\ell=j}^{\infty}\left(2^{-\ell-1}, 2^{-\ell-1}+2^{-2 \ell-2}\right)\right|}{\left|2^{-j}[-1,1]\right|}=1,
\end{aligned}
$$

the origin is a point of density for the set $F$. It follows rapidly that the origin is a point of approximate continuity of $f$.

Now we see that the function $f$ does not satisfies the condition (14) with $A=2$. Take $g \in \mathcal{D}(\mathbb{R})$ such that $g$ is non negative, with value 1 on the interval $[-1,1]$, supported on $[-2,2]$, increasing on $[-2,-1]$ and decreasing on $[1,2]$. We have the following inequalities

$$
\begin{aligned}
\lim _{j \rightarrow \infty}\left\langle\left|f\left(2^{-j} t\right)\right|, g\right\rangle & \geq \lim _{j \rightarrow \infty} \int_{-1}^{1} f\left(2^{-j} t\right) d t=\lim _{j \rightarrow \infty} 2^{j} \int_{-2^{-j}}^{2^{-j}} f(y) d y \\
& =2+\lim _{j \rightarrow \infty} 2^{j} \int_{-2^{-j}}^{2^{-j}} \sum_{\ell=0}^{\infty} 2^{\ell+2} \chi_{\left(2^{-\ell-1}, 2^{-\ell-1}+2^{-2 \ell-2}\right)}(y) d y \\
& =2+\sum_{k=0}^{\infty} 2^{-k}=4>\langle 1, g\rangle .
\end{aligned}
$$

This implies that (14) does not hold.

By a second look to this example, we can conclude that the origin is a point of approximate continuity of $f$ but the origin is not a point of Lebesgue of $f$. In addition, the origin is a point of $M^{*}$-approximate continuity of the function $\chi_{E}$ in Example 3 and the origin is not a point of Lebesgue of $\chi_{E}$. 
The condition using by Li and Zhang [32] on a neighborhood of the origin of the Fourier transform of a refinable function can be written as follows.

$$
\lim _{j \rightarrow \infty}\left|f\left(A^{-j} \mathbf{t}\right)\right|=1 \quad \text { a.e. on } G \subset \mathbb{R}^{n} \text {, some } A \text {-set. }
$$

We have already used that the condition (15) implies that the origin is a point of $(A, G)$-appriximate continuity of $f$, provided that $f(\mathbf{0})=1$, see [46, Lemma 2.5]. The converse implication may be fault as it is proved in [45, Proposition 2], where it is shown an example of a function for which the origin is a point of approximate continuity but the condition (15) does not hold when $A$ is the dyadic dilation and $G=\mathbb{R}^{n}$.

One more difference is the following. It is well-known that there exist functions $f: \mathbb{R} \rightarrow[0, \infty)$ such that

$$
\lim _{j \rightarrow \infty} f\left(4^{-j} t\right)=1, \quad \forall t \in \mathbb{R} \text { but } \lim _{j \rightarrow \infty} f\left(2^{-j} t\right) \text { does not exist. }
$$

In contrast to this, we have the following.

Proposition 1. Let $m \in\{2,3, \ldots\}$, let $A$ be an expansive linear map on $\mathbb{R}^{n}$ and let $G \subset \mathbb{R}^{n}$ be an $A$-set. Let $f: \mathbb{R}^{n} \rightarrow \mathbb{R}^{n}$ be a measurable function such that the point $\mathbf{x}$ is a point of $\left(A^{m}, G\right)$-approximate continuity of $f$. Then $\mathbf{x}$ is a point of $(A, G)$-approximate continuity of $f$.

Proof. Without loss in generality we assume that $\mathbf{x}=\mathbf{0}$. Since the origin is a point of $\left(A^{m}, G\right)$-approximate continuity of $f$, there exists an $E \subset \mathbb{R}^{n}$ such that the origin is a point of $\left(A^{m}, G\right)$-density for $E$.

Let $r>0$. We consider $j \in \mathbb{N}$ of the form $j=m p+k$ with $p \in\{0,1, \ldots\}$ and some fixed $k \in\{0, \ldots, m-1\}$. By the continuity of the linear map $A$, there exists $0<r_{k}<R_{k}$ such that $B_{r_{k}} \subset A^{-k} B_{r} \subset B_{R_{k}}$. Then we have

$$
\begin{aligned}
\frac{\left|E \cap\left[\left(G \cap A^{-(m p+k)} B_{r}\right)\right]\right|_{n}}{\left|\left(G \cap A^{-(m p+k)} B_{r}\right)\right|_{n}} & =1-\frac{\left|E^{c} \cap\left[\left(G \cap A^{-(m p+k)} B_{r}\right)\right]\right|_{n}}{\left|\left(G \cap A^{-(m p+k)} B_{r}\right)\right|_{n}} \\
& \geq 1-\frac{\left|A^{m p} E^{c} \cap\left[\left(G \cap B_{R_{k}}\right)\right]\right|_{n}}{\left|\left(G \cap B_{R_{k}}\right)\right|_{n}} \frac{\left|\left(G \cap B_{R_{k}}\right)\right|_{n}}{\left|\left(G \cap B_{r_{k}}\right)\right|_{n}} \rightarrow 1,
\end{aligned}
$$

as $p \rightarrow \infty$, because the origin is a point of $\left(A^{m}, G\right)$-density for $E$. Thus the origin is a point of $(A, G)$-density for $E$ and the statement follows.

Acknowledgement. The author would like to thank the anonymous referees for providing interesting references related to this manuscript.

\section{REFERENCES}

[1] N. Atreas, M.Papadakis, T. Stavropoulos; Extension principles for dual multiwavelet frames of $L^{2}\left(\mathbb{R}^{s}\right)$ constructed from multirefinable generators, J. Fourier Anal. Appl. 22 (2016), no. 4, 854-877.

[2] N. Atreas, A. Melas, T. Stavropoulos; Affine dual frames and extension principles. Appl. Comput. Harmon. Anal., 36 (2014) no. 1, 51-62.

[3] L.W. Baggett, H.A. Medina, K.D. Merril; Generalized multi-resolution analyses and a construction procedure for all wavelet sets in $\mathbb{R}^{n}$, J. Fourier Anal. Appl., 5 (1999), 563-573.

[4] D. Bakić; Semi-orthogonal parseval frame wavelets and generalized multiresolution analyses, Appl. Comput. Harmon. Anal. 21 (2006), no. 3, 281-304

[5] J.J. Benedetto, S. Li; The theory of multiresolution analysis frames and applications to filter banks, Appl. Comput. Harmon. Anal. 5 (1998), 389-427. 
[6] J.J. Benedetto, O.M. Treiber; Wavelet frames: Multiresolution analysis and extension principles, L. Debnath (Ed.), Wavelet Transforms and TimeFrequency Signal Analysis, Birkhuser (2001), pp. 336.

[7] M. Bownik; Riesz wavelets and generalized multiresolution analyses, Appl. Comput. Harmon. Anal. 14 (2003), no. 3, 181-194.

[8] M. Bownik, G. Garrigós; Biorthogonal wavelets, MRA's and shift-invariant spaces, Studia Math. 160 (2004), no. 3, 231-248.

[9] M. Bownik, Z. Rzeszotnik; Construction and reconstruction of tight framelets and wavelets via matrix mask functions, J. Funct. Anal. 256 (2009), no. 4, 1065-1105.

[10] C. de Boor, R.A. DeVore, A. Ron; On the construction of multivariate (pre)wavelets, Constructive Approximation 9 (1993), No.2-3, 123-166.

[11] A. Bruckner; Differentiation of real functions, Lecture Notes in Mathematics, 659. Springer, Berlin, 1978.

[12] A. Calogero, G. Garrigós; A characterization of wavelet families arising from biorthogonal MRA's of multiplicity d, J. Geom. Anal. 11 (2001), no. 2, 187-217.

[13] O. Christensen; An introduction to frames and Riesz bases, Birkhäser, Boston, 2003.

[14] C.K. Chui, W. He, J. Stöckler; Compactly supported tight and sibling frames with maximum vanishing moments, Appl. Comput. Harmonic Anal. 13 (2002), 224-262.

[15] C.K. Chui, W. He, J. Stöckler, Q. Sun; Compactly supported tight affine frames with integer dilations and maximum vanishing moments, Adv. Comput. Math. 18 (2003), no. 2-4, 159187.

[16] X. Dai, Y. Diao, Q. Gu, D. Han; Frame wavelets in subspaces of $L^{2}\left(\mathbb{R}^{d}\right)$, Proc. Am. Math. Soc. 130 (2002), No. 11, 3259-3267.

[17] I. Daubechies, B. Han, A. Ron, Z.W. Shen; Framelets: MRA-based constructions of wavelet frames, Appl. Comput. Harmon. Anal. 14 (2003), 1-46.

[18] R. Duffin, A. Schaeffer; A class of nonharmonic Fourier series, Trans. Amer. Math. Soc. 72 (1952), 341-366.

[19] G. Gripenberg; A necessary and sufficient condition for the existence of a father wavelet, Studia Math. 114 (1995), no. 3, 207-226.

[20] K. Gröchening, W.R. Madych; Multiresolution analysis, Haar bases and self-similar tillings of $R^{n}$, IEEE Trans. Inform. Theory, 38(2), (1992) 556-568.

[21] B. Han Nonhomogeneous wavelet systems in high dimensions, App. Comput. Harmon. Anal. 32 (2012), no. 2, 169-196.

[22] B. Han; Pairs of frequency-based nonhomogeneous dual wavelet frames in the distribution space, Appl. Comput. Harmon. Anal. 29 (2010), no. 3, 330-353.

[23] B. Han; On dual wavelet tight frames, Applied Comput. Harmon. Anal. 4 (1997), 380-413.

[24] E. Hernández, G. Weiss; A first course on Wavelets, CRC Press Inc., 1996.

[25] H.-F. Jia, Y.-Z. Li; Refinable function-based construction of weak (quasi-)affine bi-frames, J. Fourier Anal. Appl. 20 (2014), no. 6, 1145-1170.

[26] R.Q. Jia, Z. Shen; Multiresolution and Wavelets, Proc. Edinburgh Math. Soc. (2) 37 (1994), no. 2, 271-300.

[27] K.S. Kazarian, A. San Antolín; Characterization of scaling functions in a frame multiresolution analysis in $H_{G}^{2}$, Topics in classical analysis and applications in honor of Daniel Waterman. Hackensack, NJ: World Scientific. 118-140 (2008).

[28] H.O. Kim, R.Y. Kim, Y.H. Lee, J.K. Lim; On Riesz wavelets associated with multiresolution analyses, Appl. Comput. Harmon. Anal. 13 (2002), no. 2, 138-150.

[29] H.O. Kim, R.Y. Kim, J.K. Lim; Characterizations of biorthogonal wavelets which are associated with biorthogonal multiresolution analyses, Appl. Comput. Harmon. Anal. 11 (2001), no. 2, 263-272.

[30] A. Krivoshein, V. Protasov, M. Skopina; Multivariate wavelet frames, Industrial and Applied Mathematics. Springer, Singapore, 2016.

[31] Y.-Z. Li, Q.-F. Lian; Reducing subspace frame multiresolution analysis and frame wavelets Commun. Pure Appl. Anal. 6 (2007), no. 3, 741-756.

[32] Y.-Z. Li, J.P. Zhang; Extension principles for affine dual frames in reducing subspaces, Appl. Comput. Harmon. Anal. 46 (2019), no. 1, 177-191.

[33] Y.-Z. Li, L. Zhang; An embedding theorem on reducing subspace frame multiresolution analysis, Kodai Math. J. 35 (2012), no. 1, 157-172. 
[34] Y.-Z. Li, F.-Y. Zhou; Generalized multiresolution structures in reducing subspaces of $L^{2}\left(\mathbb{R}^{d}\right)$, Sci. China Math. 56 (2013), no. 3, 619-638.

[35] Y.-Z., Li, F.-Y. Zhou; GMRA-based construction of framelets in reducing subspaces of $L^{2}\left(\mathbb{R}^{d}\right)$, Int. J. Wavelets Multiresolut. Inf. Process. 9 (2011), no. 2, 237-268.

[36] S. Mallat; Multiresolution approximations and wavelet orthonormal bases for $L^{2}(R)$, Trans. Amer. Math. Soc. 315 (1989), 69-87.

[37] Y. Meyer; Ondelettes et opérateurs. I, Hermann, Paris, 1996 [English Translation: Wavelets and operators, Cambridge University Press, 1992]

[38] I.P. Nathanson; Theory of functions of a real variable, London, vol. II, 1960.

[39] M. Papadakis; Frames of translates in abstract Hilbert spaces and the generalized frame multiresolution analysis, Trends in approximation theory (Nashville, TN, 2000), 353362, Innov. Appl. Math., Vanderbilt Univ. Press, Nashville, TN, 2001.

[40] M. Papadakis; On the dimension function of orthonormal wavelets, Proc. Amer. Math. Soc. 128 (2000), no. 7, 20432-049.

[41] A. Petukhov; Explicit construction of framelets, Appl. Comput. Harmon. Anal. 11 (2001), no. 2, 313-327.

[42] A. Ron, Z.W Shen; Affine systems in $L^{2}\left(\mathbb{R}^{d}\right)$. II. Dual systems, Dedicated to the memory of Richard J. Duffin. J. Fourier Anal. Appl. 3 (1997), no. 5, 617-637.

[43] A. Ron, Z.W. Shen; Affine systems in $L^{2}\left(\mathbb{R}^{d}\right)$ : The analysis of the analysis operator, J. Funct. Anal. 148 (1997), 408-447.

[44] A. San Antolín; On translation invariant multiresolution analysis, Glas. Mat. Ser. III 49(69) (2014), no. 2, 377-394.

[45] A. San Antolín, Characterization of low pass filters in a multiresolution analysis, Studia Math. 190 (2009), 99-116.

[46] M. Soto-Bajo; Closure of dilates of shift-invariant subspaces, Cent. Eur. J. Math. 11 (2013), no. $10,1785-1799$

[47] T. Stavropoulos; The geometry of extension principles, Houston J. Math. 38, no. 3, 833-853 (2012).

[48] R.A. Zalik; Riesz bases and multiresolution analyses, Appl. Comput. Harmon. Anal. 7 (1999), no. $3,315-331$.

Departamento de Matemáticas, Universidad de Alicante, 03080 Alicante, Spain.

E-mail address: angel.sanantolin@ua.es 